Check for updates

Cite this: RSC Chem. Biol., 2020, 1,60

Received 2nd February 2020, Accepted 16th May 2020

DOI: 10.1039/d0cb00012d

rsc.li/rsc-chembio

\section{Biological evaluation of native streptococcal competence stimulating peptides reveals potential crosstalk between Streptococcus mitis and Streptococcus pneumoniae and a new scaffold for the development of $S$. pneumoniae quorum sensing modulators $\dagger$}

\begin{abstract}
Tahmina Ahmed Milly and Yftah Tal-Gan (D) *
Streptococcus pneumoniae, an opportunistic human pathogen, acquires genes from its neighboring species of the mitis group of streptococci, which confer antibiotic resistances and allow it to produce diverse virulence factors. Most species of the mitis group are naturally competent, and they utilize the competence stimulating peptide (CSP) and the CSP-dependent competence regulon, a conserved quorum sensing (QS) circuit, to regulate their competence behavior. The dependence of the mitis group on this communication pathway makes QS a potential target to control their behavior. In this work, we sought to evaluate the impact of the native pheromones of the adjacent species of $S$. pneumoniae to modulate the activity of the $S$. pneumoniae competence regulon. Our results revealed the potential role of S. mitis as a modulator of QS in S. pneumoniae. Most importantly, our analysis also revealed that by using the native pheromone of $S$. mitis as a template, highly potent pan-group agonists and antagonists of the pneumococcal competence regulon could be developed. The newly developed QS modulators may have therapeutic utility in treating pneumococcal infections.
\end{abstract}

Streptococcus pneumoniae (pneumococcus), a member of the mitis group of streptococci, is an opportunistic human pathogen that is a major cause of a variety of diseases, including the deadly pneumonia, bacteremia, meningitis, sepsis and otitis media. ${ }^{1}$ It has been estimated that more than 22000 deaths and 445000 hospitalizations, with a $\$ 3.5$ billion direct medical cost, occur annually in the United States as a result of pneumococcal infections. ${ }^{2}$ Furthermore, the increasing rate of resistance development against several antibiotics, including vancomycin, quinolones, macrolides and beta lactams by the recombinogenic pneumococcal strains necessitates the development of alternative strategies to attenuate pneumococcal infections. ${ }^{3,4}$ Pneumococcus utilizes the competence regulon, a conserved quorum sensing (QS) circuit (Fig. 1), to acquire genetic material from the neighboring environment, including genes that confer antibiotic resistance, as well as to regulate a wide range of functions related to pathogenicity such as

Department of Chemistry, University of Nevada, Reno, 1664 N. Virginia Street, Reno, Nevada 89557, USA. E-mail: ytalgan@unr.edu

$\dagger$ Electronic supplementary information (ESI) available: Full details of experimental procedures, peptide characterization, initial screening results, and doseresponse curves for all CSP analogues (PDF). See DOI: 10.1039/d0cb00012d

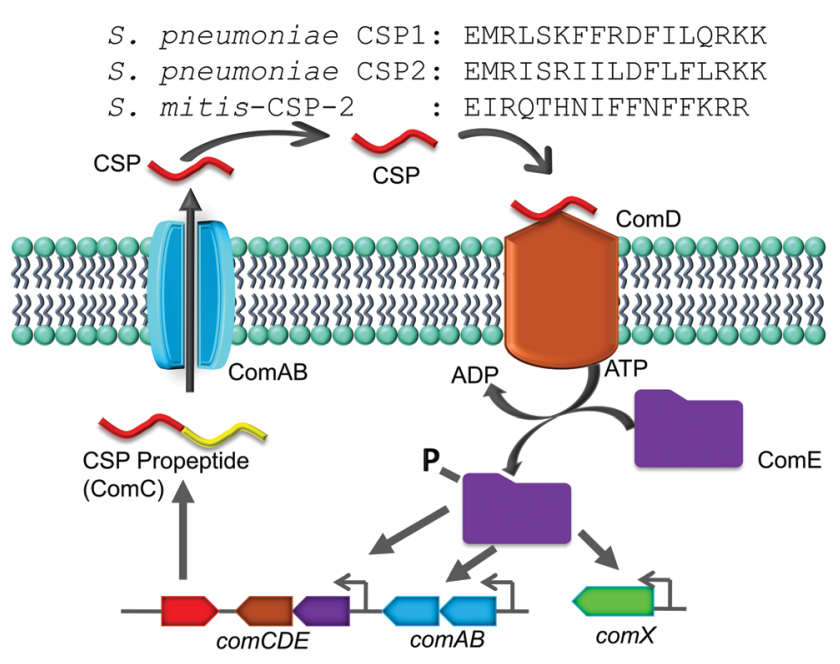

Fig. 1 CSP-mediated QS circuit of the mitis group of streptococci. The CSP propeptide, ComC, is processed and secreted by the ComAB transporter as the mature peptide, CSP, which interacts with the cognate receptor, ComD. Activated ComD phosphorylates ComE, which then autoactivates the competence QS circuit. The sequences for S. pneumoniae CSP1, S. pneumoniae CSP2 and S. mitis-CSP-2 are shown at the top. 
virulence factor production and biofilm formation. ${ }^{5-9}$ Thus, intercepting this QS circuitry could be utilized as a potential non-antibiotic therapeutic strategy to control pneumococcal infections without affecting the survival of the bacteria, thus minimizing the selective pressure for resistance development. ${ }^{10-16}$

The competence regulon in pneumococcus relies on the production of, secretion of and response to a 17-amino-acid competence stimulating peptide (CSP, Fig. 1) signal. ${ }^{5}$ Different strains of pneumococcus can produce different CSP signals and the two major forms of CSP (CSP1 and CSP2) share 50\% homology with each other. ${ }^{17}$ Each CSP signal is associated with a cognate transmembrane histidine kinase receptor, termed ComD (ComD1 and ComD2, respectively). ${ }^{18}$ The competence regulon QS circuitry (Fig. 1) involves the secretion of mature CSP signal into the extracellular environment with the help of a dedicated $\mathrm{ABC}$ transporter, ComAB. ${ }^{5}$ As the bacteria grow, the concentration of CSP increases, and once a threshold concentration is attained, the CSP pheromone can bind to and activate the ComD receptor. ${ }^{8}$ ComD activation prompts the phosphorylation of the cytoplasmic response regulator, ComE, which then acts as a transcription factor to upregulate the transcription of the QS circuitry genes (comABCDE) as well as the $\operatorname{com} X$ gene, the master regulator of the QS circuitry that controls the different QS-regulated phenotypes. ${ }^{6-8}$

Similar to $S$. pneumoniae, other species of the mitis and the anginosus streptococcal groups are naturally competent. Regardless of the specific CSP pheromone involved, these species utilize a similar pheromone-dependent competence regulon to upregulate the expression of competence genes required for DNA uptake and recombination. ${ }^{19}$ In the anginosus group, different species utilize the same CSP signaling molecule, while a large variation of CSP pheromones have been reported within the same species in the mitis group, specifically among Streptococcus mitis strains. ${ }^{20-22}$ The mitis group of streptococci is composed of 13 species; most of them are commensal bacteria, with $S$. pneumoniae also being a notorious opportunistic pathogen. $^{20,21}$ The recombination events among the mitis group of streptococci have been shown to be instrumental in the development of antibiotic resistance. Specifically, interspecies gene transfer of both antimicrobial resistance and virulence genes was observed between $S$. pneumoniae and its neighboring species, $S$. mitis, which shares more than $80 \%$ of its genes with $S$. pneumoniae. ${ }^{21,23}$ In this study we aimed to evaluate the impact that native CSP signals of streptococci of the mitis and anginosus groups have on the activity of the S. pneumoniae competence regulon. To this end, we chemically synthesized different reported native streptococcal CSP pheromones of the mitis and anginosus groups and tested their ability to activate/inhibit the $S$. pneumoniae ComD1 and ComD2 receptors. Our initial analysis revealed that one of the CSP pheromones of $S$. mitis, $S$. mitis-CSP-2 (Fig. 1), can activate both $S$. pneumoniae ComD receptors. We therefore chose S. mitisCSP-2 as the lead scaffold for the development of pan-group $S$. pneumoniae QS modulators. Indeed, using $S$. mitis-CSP-2 as a template, we were able to develop potent pan group QS inhibitors of $S$. pneumoniae QS. Moreover, our results revealed potential crosstalk between $S$. mitis and $S$. pneumoniae.

\section{Results and discussion}

\section{Synthesis and initial screening of native streptococcal competence pheromones (CSPs)}

In this work, we first set out to evaluate potential crosstalk between species of the mitis group by testing the ability of native streptococcal competence pheromones (CSPs) to modulate the activity of the $S$. pneumoniae competence regulon QS circuitry. To this end, we chemically synthesized different native CSPs of the most common commensal bacterial strains of the mitis group (see Table 1 for strain information and CSP sequences). To explore potential crosstalk between the different groups of streptococci, we decided to include CSP pheromones from the anginosus group. Since most of the species of the anginosus group, namely $S$. anginosus, $S$. constellatus and $S$. intermedius, produce the same CSP signal, ${ }^{21}$ we synthesized only one CSP signal (S. intermedius-CSP) representing the anginosus group of

Table 1 Sequence of the streptococcal competence pheromones (CSPs) from select strains of streptococci for which the pheromone has been synthesized in this study

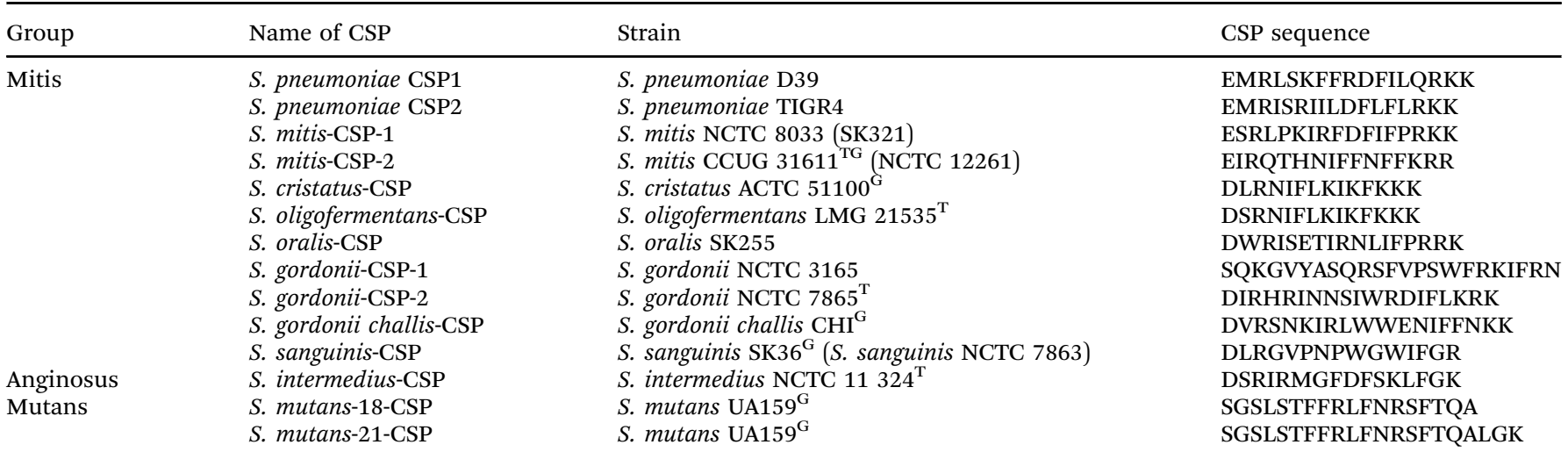

${ }^{\mathrm{T}}$ Type strain. ${ }^{\mathrm{G}}$ Genome sequence available. 
streptococci (Table 1). Lastly, in the initial screening assay, we included the two CSP forms of $S$. mutans, $S$. mutans-21-CSP (pre-mature extracellular signal) and $S$. mutans-18-CSP (mature extracellular signal), ${ }^{24}$ to test for potential crosstalk between opportunistic pathogens. All of the CSP pheromones (Table 1) were constructed using established solid-phase peptide synthesis (SPPS) protocols on 4-benzyloxybenzyl alcohol (Wang) resin. ${ }^{25}$ The peptides were then cleaved and purified to homogeneity using semipreparative RP-HPLC ( $>95 \%$ purity), and their identity was confirmed using mass spectrometry (see the Experimental section and ESI $\dagger$ for full experimental details).

Next, we tested the ability of the native streptococcal CSPs to modulate the activity of the $S$. pneumoniae ComD receptors (both ComD1 and ComD2) using a $\beta$-galactosidase cell-based bacterial reporter assay. Two $\beta$-gal reporter strains, D39pcomX::lacZ and TIGR4pcomX::lacZ, previously constructed by Lau and co-workers, were utilized to evaluate the QS modulation in both groups of $S$. pneumoniae. ${ }^{26}$ These reporter systems carry the lac $Z$ gene under the control of the $\operatorname{com} X$ promoter; thus, upon QS activation, lacZ will be expressed, allowing the monitoring of the QS response by measuring the $\beta$-galactosidase activity (see the Experimental section for details). We then conducted an initial screening of all the native CSP pheromones at high peptide concentration $(10 \mu \mathrm{M}$; see Fig. S-1, S-6, S-9, and S-14, ESI $\dagger)$. The initial screening results revealed that of the twelve CSP signals tested, only one native CSP pheromone, $S$. mitis-CSP-2, was able to fully activate both the $S$. pneumoniae ComD receptors $(>75 \%$ activation compared to the native $S$. pneumoniae CSPs; Fig. S-1 and S-9, $\mathrm{ESI} \dagger)$. The $\mathrm{EC}_{50}$ values of this peptide were determined through dose response curves and found to be $663 \mathrm{nM}$ and $635 \mathrm{nM}$ when activating the $S$. pneumoniae ComD1 and ComD2 receptors, respectively (Table 2). Although these $\mathrm{EC}_{50}$ values are 12 - to 64-fold higher than the native pneumococcus CSPs, the values are still biologically relevant as the native CSP concentrations in streptococci supernatants were found to be in the micromolar range. ${ }^{15}$ Since none of the other native peptides were capable of activating/inhibiting the $S$. pneumoniae QS circuitry, we selected the $S$. mitis-CSP-2 pheromone as a template for the development of pan-group $S$. pneumoniae QS modulators that could be utilized to attenuate pneumococcal infections.

\section{Point modification S. mitis-CSP-2 analogues}

Previously, the Tal-Gan Laboratory had conducted a detailed structure-activity relationship (SAR) analysis of the two $S$. pneumoniae signal variants, CSP1 and CSP2, against the two $S$. pneumoniae ComD receptors, ComD1 and ComD2, and identified the critical residues involved in ComD1 and ComD2 receptor binding and activation. ${ }^{27-29}$ Based on the SAR results of both the CSP1 and CSP2 scaffolds in S. pneumoniae, we designed ten $S$. mitis-CSP-2 analogues bearing a point modification in key positions to match the corresponding residue in $S$. pneumoniae CSP1 or CSP2 pheromones and examined their ability to modulate the competence regulon of $S$. pneumoniae (Table 2). Since $S$. mitis-CSP-2 and the two CSP signals of $S$. pneumoniae differ in many positions (Fig. 1 and Table 1), we chose the key positions contributing to the $S$. pneumoniae receptor binding and activation, all of which are in the $N$-terminus and the central region of $S$. pneumoniae CSPs, and systematically replaced each residue in S. mitis-CSP-2 with the residues found in $S$. pneumoniae CSP1 or CSP2. We excluded the modifications related to the $C$-terminus of the pheromones as the $C$-terminal residues of $S$. pneumoniae CSP1 and CSP2 were found to be dispensable. ${ }^{27}$

The initial screening of the $S$. mitis-CSP-2 point-modification analogues revealed that most of the mutated analogues exhibited higher potency against both the $S$. pneumoniae ComD1 and ComD2 receptors compared to the native $S$. mitisCSP-2 (Fig. S-2 and S-10, (ESI $\dagger$ ) and Table 2). Specifically, this analysis revealed that $S$. mitis-CSP-2-I2M, a point-mutated analogue that introduced the conserved residue, Met2, that is present in both the $S$. pneumoniae pheromones, has increased potency against both the $S$. pneumoniae ComD receptors compared to the native $S$. mitis-CSP-2. These results support previous observations that Met2 plays a critical role in binding and activation in

Table $2 \mathrm{EC}_{50}$ values of the S. mitis-CSP-2 point-modification analogues against the S. pneumoniae ComD1 and ComD2 receptors ${ }^{a}$

\begin{tabular}{|c|c|c|c|c|c|}
\hline \multirow[b]{2}{*}{ Peptide name } & \multirow[b]{2}{*}{ Peptide sequence } & \multicolumn{2}{|c|}{ S. pneumoniae ComD1 } & \multicolumn{2}{|c|}{ S. pneumoniae ComD2 } \\
\hline & & $\mathrm{EC}_{50}^{b}(\mathrm{nM})$ & $95 \% \mathrm{CI}^{c}$ & $\mathrm{EC}_{50}^{b}(\mathrm{nM})$ & $95 \% \mathrm{CI}^{c}$ \\
\hline S. pneumoniae $\mathrm{CSP}^{d}$ & EMRLSKFFRDFILQRKK & 10.3 & $6.27-16.8$ & 526 & $498-556$ \\
\hline S. pneumoniae $\mathrm{CSP}^{d}$ & EMRISRIILDFLFLRKK & 1650 & $1190-2300$ & 50.7 & $40.6-63.2$ \\
\hline S. mitis-CSP-2 & EIRQTHNIFFNFFKRR & 663 & $608-722$ & 635 & $426-947$ \\
\hline S. mitis-CSP-2-I2M & EMRQTHNIFFNFFKRR & 87.7 & $79.1-97.3$ & 136 & $89.0-208$ \\
\hline S. mitis-CSP-2-F10D & EIRQTHNIFDNFFKRR & $-^{e}$ & - & $>1000$ & - \\
\hline S. mitis-CSP-2-Q4L & EIRLTHNIFFNFFKRR & 88.2 & $64.6-121$ & 252 & $231-275$ \\
\hline S. mitis-CSP-2-N7F & EIRQTHFIFFNFFKRR & 140 & $113-172$ & 471 & $459-482$ \\
\hline S. mitis-CSP-2-I8F & EIRQTHNFFFNFFKRR & 128 & $93.2-176$ & 209 & $139-313$ \\
\hline S. mitis-CSP-2-N11F & EIRQTHNIFFFFFKRR & 4.63 & $2.43-8.84$ & 220 & $194-248$ \\
\hline S. mitis-CSP-2-F12I & EIRQTHNIFFNIFKRR & $-^{e}$ & - & $>1000$ & - \\
\hline S. mitis-CSP-2-Q4I & EIRITHNIFFNFFKRR & $-^{e}$ & - & - & - \\
\hline S. mitis-CSP-2-N7I & EIRQTHIIFFNFFKRR & 101 & $79.5-129$ & 211 & $147-303$ \\
\hline S. mitis-CSP-2-F12L & EIRQTHNIFFNLFKRR & 54.3 & $34.7-84.7$ & 813 & $539-1230$ \\
\hline
\end{tabular}

${ }^{a}$ See the Experimental section for full experimental details and the (ESI) for plots of agonism dose response curves. ${ }^{b}$ EC $_{50}$ values were determined by testing peptides over a range of concentrations. ${ }^{c} 95 \%$ confidence interval. ${ }^{d} \mathrm{EC}_{50}$ values of $S$. pneumoniae $\mathrm{CSP}$ and $\mathrm{CSP} 2$ from ref. $27 .{ }^{e} \mathrm{EC}_{50}$ not determined due to the analogue's low induction in the primary agonism screening assay. 
both the $S$. pneumoniae receptors. ${ }^{27,30}$ On the contrary, the second $S$. mitis-CSP-2-based analogue bearing a conserved residue present in both $S$. pneumoniae CSPs, $S$. mitis-CSP-2-F10D, was found to activate only the $S$. pneumoniae ComD2 receptor, although only at a high concentration ( $>1000 \mathrm{nM}$, Table 2). Looking at the $S$. pneumoniae CSP1-based modifications, alterations of the hydrophobic residues in the central region of $S$. mitis at positions 4, 7, 8, 11 and 12 in $S$. mitis-CSP-2 with the corresponding residues in $S$. pneumoniae CSP1 revealed that, with the exception of $S$. mitisCSP-2-F12I, all the resulting analogues exhibit enhanced activity against ComD1 compared to the native $S$. mitis-CSP-2. Importantly, the replacement of the hydrophilic residue Asn at position 11 in $S$. mitis-CSP-2 with the hydrophobic residue Phe resulted in an analogue ( $S$. mitis-CSP-2-N11F) with $>140$-fold increased potency compared to the native $S$. mitis-CSP-2 signal against the S. pneumoniae ComD1 receptor, exhibiting comparable activity to the pneumococcal native peptide, S. pneumoniae CSP1. With the exception of $S$. mitis-CSP-2-F12I, all the resulting analogues also exhibited increased potency compared to the native $S$. mitis-CSP-2 signal against the $S$. pneumoniae ComD2 receptor (Table 2).

Moving to the $S$. pneumoniae CSP2-based modifications (Q4I, N7I, F12L), the initial screening revealed that only $S$. mitis-CSP-2-N7I exhibits enhanced potency compared to native S. mitis-CSP-2 against both pneumococcal ComD receptors, highlighting the important role the hydrophobic residues play in both pneumococcal receptor binding and activation. On the contrary, $S$. mitis-CSP-2-Q4I was found to be relatively inactive against both the $S$. pneumoniae ComD receptors (Table 2).

\section{Multiple modifications $S$. mitis-CSP-2 analogues}

The point modification study revealed several important $S$. mitis-CSP-2-based S. pneumoniae QS modulators. We therefore set out to utilize the recently acquired SAR insight to rationally design $S$. pneumoniae QS modulators with enhanced potencies. To this end, we synthesized a library of double and triple modification analogues where we incorporated multiple beneficial point modifications into a single analogue. Expectedly, the initial evaluation revealed that most of the doubly modified analogues are more active than the native $S$. mitis-CSP-2 signal against both $S$. pneumoniae ComD receptors. However, while some dual-modified analogues exhibited slightly increased or similar potency relative to the single replacement analogues, other double modification analogues resulted in reduction in potency compared to the single point modification analogues (Table 3). Specifically, the combination of the Q4L modification with other central region hydrophobic modifications (N7F, N7I, $\mathrm{I} 8 \mathrm{~F}$, or $\mathrm{N} 11 \mathrm{~F}$ ) resulted in doubly-modified analogues with reduced potency compared to the monosubstituted analogues against both pneumococcal ComD receptors. Most importantly, we identified one double modification analogue, $S$. mitis-CSP-2N7II8F, that can bind and activate both pneumococcal ComD receptors with enhanced potencies compared to the single replacement analogues (compare the $\mathrm{EC}_{50}$ values of $S$. mitisCSP-2-N7I and $S$. mitis-CSP-2-I8F against the pneumococcal ComD1 and ComD2 receptors in Table 2 with the $\mathrm{EC}_{50}$ values of $S$. mitis-CSP-2-N7II8F against the pneumococcal ComD1 and
ComD2 receptors in Table 3), making it the most potent $S$. mitisCSP-2-based pan-group pneumococcal QS activator identified in the double modification library. Interestingly, a similar doublemodification where Asn7 was replaced with phenylalanine, the native residue in $S$. pneumoniae CSP1, resulted in a doublemodified analogue, $S$. mitis-CSP-2-N7FI8F, that lost its activity against the pneumococcal ComD1 receptor, while maintaining its activity against the pneumococcal ComD2 receptor (Table 3).

Moving to the triple-modified analogues, it appears that the majority of combinations were not tolerated well, as most analogues exhibited similar or reduced activities compared to the single- or doubly-modified analogues against the pneumococcal ComD receptors (Table 3). However, the triple-modified library also revealed four $S$. mitis-CSP-2-based analogues, S. mitis-CSP-2-I2MI8FN11F, S. mitis-CSP-2-I2MN7II8F, S. mitisCSP-2-I2MQ4LI8F, and $S$. mitis-CSP-2-I2MN7FI8F, with activities at the low nanomolar range $\left(\mathrm{EC}_{50}\right.$ values $\left.<100 \mathrm{nM}\right)$ against both pneumococcal ComD receptors (Table 3). All four analogues share the same two modifications, I2M and I8F, suggesting that these two modifications are critical for pangroup reactivity of the $S$. mitis-CSP-2 scaffold against the pneumococcus ComD receptors.

\section{Development of $S$. mitis-CSP-2-based pneumococcal pan-group QS inhibitors}

It has been shown that the interception of the pneumococcal competence regulon by either targeting CSP export or disrupting the native CSP:ComD interactions can be utilized to attenuate QS-dependent pneumococcus pathogenicity. ${ }^{26,27,31-33}$ All the previously developed CSP-based inhibitors share the same key modification, the replacement of glutamic acid at position 1 with alanine. $^{26-29,32-35}$ We therefore reasoned that the $S$. mitis-CSP-2 template can also be converted into a pneumococcus ComD inhibitor by incorporating the same E1A modification. To this end, we first replaced Glu1 with alanine in the native $S$. mitisCSP-2 to afford $S$. mitis-CSP-2-E1A and found that the resulting analogue can effectively inhibit the pneumococcus ComD1 receptor, albeit at a 6-fold higher concentration than $S$. pneumoniae CSP1-E1A ( IC $_{50}$ value of $497 \mathrm{nM}$ ), while losing all activity against the S. pneumoniae ComD2 receptor (Table 4).

Next, we selected the top $S$. mitis-CSP-2 modified analogues identified in this study and incorporated the E1A modification. We hypothesized that combining these modifications together with the E1A mutation would result in $S$. mitis-CSP-2-based analogues that maintain the binding affinity to the pneumococcal ComD receptors but may not be able to activate the receptors, leading to competitive pan-group antagonists of the $S$. pneumoniae competence regulon. To test our hypothesis, we synthesized eleven $S$. mitis-CSP-2 analogues bearing the E1A modification. These analogues included two $S$. mitis-CSP-2 singlemodified analogues, five $S$. mitis-CSP-2 double-modified analogues and four $S$. mitis-CSP-2 triple-modified analogues, all bearing also the E1A modification, and tested their ability to modulate the QS circuitry in both pneumococcal specificity groups (Table 4). Our analysis revealed that all the $S$. mitis-CSP-2-E1A-based analogues that had the N11F modification were inactive against 
Table $3 \mathrm{EC}_{50}$ values of the S. mitis-CSP-2 multiple-modification analogues against the S. pneumoniae ComD1 and ComD2 receptors ${ }^{a}$

\begin{tabular}{|c|c|c|c|c|c|}
\hline \multirow[b]{2}{*}{ Peptide name } & \multirow[b]{2}{*}{ Peptide sequence } & \multicolumn{2}{|c|}{ S. pneumoniae ComD1 } & \multicolumn{2}{|c|}{ S. pneumoniae ComD2 } \\
\hline & & $\mathrm{EC}_{50}^{b}(\mathrm{nM})$ & $95 \% \mathrm{CI}^{c}$ & $\mathrm{EC}_{50}^{b}(\mathrm{nM})$ & $95 \% \mathrm{CI}^{c}$ \\
\hline S. pneumoniae $\mathrm{CSP}^{d}{ }^{d}$ & EMRLSKFFRDFILQRKK & 10.3 & $6.27-16.8$ & 526 & $498-556$ \\
\hline S. pneumoniae $\operatorname{CSP} 2^{d}$ & EMRISRIILDFLFLRKK & 1650 & $1190-2300$ & 50.7 & $40.6-63.2$ \\
\hline S. mitis-CSP-2 & EIRQTHNIFFNFFKRR & 663 & $608-722$ & 635 & $426-947$ \\
\hline S. mitis-CSP-2-I2MQ4L & EMRLTHNIFFNFFKRR & 49.9 & $42.4-58.9$ & 141 & $76.8-257$ \\
\hline S. mitis-CSP-2-I2MN7F & EMRQTHFIFFNFFKRR & 131 & $76.5-225$ & 123 & $91.7-166$ \\
\hline S. mitis-CSP-2-I2MN7I & EMRQTHIIFFNFFKRR & 448 & $345-582$ & 54.8 & $51.7-58.0$ \\
\hline S. mitis-CSP-2-I2MI8F & EMRQTHNFFFNFFKRR & 151 & $87.1-262$ & 83.2 & 65.9-105 \\
\hline S. mitis-CSP-2-I2MN11F & EMRQTHNIFFFFFKRR & 17.7 & $11.5-27.4$ & 753 & $573-990$ \\
\hline S. mitis-CSP-2-I2MF12L & EMRQTHNIFFNLFKRR & 25.8 & $13.1-50.8$ & 146 & $81.3-261$ \\
\hline S. mitis-CSP-2-Q4LN7F & EIRLTHFIFFNFFKRR & 371 & $293-469$ & 322 & $257-402$ \\
\hline S. mitis-CSP-2-Q4LN7I & EIRLTHIIFFNFFKRR & 348 & $278-436$ & 315 & $229-433$ \\
\hline S. mitis-CSP-2-Q4LI8F & EIRLTHNFFFNFFKRR & 280 & $153-513$ & 370 & $275-499$ \\
\hline S. mitis-CSP-2-Q4LN11F & EIRLTHNIFFFFFKRR & 9.38 & $8.44-10.4$ & 434 & $308-609$ \\
\hline S. mitis-CSP-2-Q4LF12L & EIRLTHNIFFNLFKRR & 68.7 & $50.6-93.2$ & 202 & $193-211$ \\
\hline S. mitis-CSP-2-N7FI8F & EIRQTHFFFFNFFKRR & $-^{e}$ & - & 24.6 & $21.9-27.6$ \\
\hline S. mitis-CSP-2-N7FN11F & EIRQTHFIFFFFFKRR & $-^{e}$ & - & $-^{e}$ & - \\
\hline S. mitis-CSP-2-N7FF12L & EIRQTHFIFFNLFKRR & 156 & $84.9-287$ & 533 & $262-1083$ \\
\hline S. mitis-CSP-2-N7II8F & EIRQTHIFFFNFFKRR & 87.2 & $56.8-134$ & 22.8 & $13.1-40.0$ \\
\hline S. mitis-CSP-2-N7IN11F & EIRQTHIIFFFFFKRR & $-^{e}$ & - & $-^{e}$ & - \\
\hline S. mitis-CSP-2-N7IF12L & EIRQTHIIFFNLFKRR & 613 & $506-743$ & 321 & $216-475$ \\
\hline S. mitis-CSP-2-I8FN11F & EIRQTHNFFFFFFKRR & 12.2 & $5.73-26.0$ & 284 & $188-429$ \\
\hline S. mitis-CSP-2-I8FF12L & EIRQTHNFFFNLFKRR & 160 & $104-248$ & 202 & $145-281$ \\
\hline S. mitis-CSP-2-N11FF12L & EIRQTHNIFFFLFKRR & 4.97 & $4.12-5.99$ & 127 & $117-137$ \\
\hline S. mitis-CSP-2-I2MQ4LN7F & EMRLTHFIFFNFFKRR & 137 & $105-178$ & 75.6 & $69.2-82.7$ \\
\hline S. mitis-CSP-2-I2MI8FN11F & EMRQTHNFFFFFFKRR & 6.95 & $4.69-10.3$ & 26.2 & $14.1-49.0$ \\
\hline S. mitis-CSP-2-I2MN7FF12L & EMRQTHFIFFNLFKRR & 72.8 & $46.7-114$ & 112 & $76.4-163$ \\
\hline S. mitis-CSP-2-I2MN7IISF & EMRQTHIFFFNFFKRR & 61.6 & $46.1-82.3$ & 2.67 & $1.91-3.73$ \\
\hline S. mitis-CSP-2-I2MQ4LF12L & EMRLTHNIFFNLFKRR & 17.1 & $15.8-18.5$ & 139 & $92.6-210$ \\
\hline S. mitis-CSP-2-I2MQ4LI8F & EMRLTHNFFFNFFKRR & 42.0 & $25.7-68.5$ & 30.0 & $15.1-59.3$ \\
\hline S. mitis-CSP-2-I2MQ4LN7I & EMRLTHIIFFNFFKRR & 155 & $141-170$ & 187 & $150-233$ \\
\hline S. mitis-CSP-2-I2MQ4LN11F & EMRLTHNIFFFFFKRR & 14.8 & $11.3-19.4$ & 188 & $126-281$ \\
\hline S. mitis-CSP-2-Q4LN7FI8F & EIRLTHFFFFNFFKRR & 427 & $402-454$ & 87.2 & $69.1-110$ \\
\hline S. mitis-CSP-2-Q4LN7II8F & EIRLTHIFFFNFFKRR & 242 & $120-489$ & $-^{e}$ & - \\
\hline S. mitis-CSP-2-Q4LN7FN11F & EIRLTHFIFFFFFKRR & $-^{e}$ & - & $-^{e}$ & - \\
\hline S. mitis-CSP-2-I2MN7FI8F & EMRQTHFFFFNFFKRR & 63.6 & $34.1-119$ & 13.5 & $11.7-15.5$ \\
\hline S. mitis-CSP-2-Q4LN7FF12L & EIRLTHFIFFNLFKRR & 426 & $292-622$ & $-^{e}$ & - \\
\hline S. mitis-CSP-2-N7FI8FF12L & EIRQTHFFFFNLFKRR & 101 & $63.5-160$ & 67.6 & $36.2-126$ \\
\hline S. mitis-CSP-2-N7II8FN11F & EIRQTHIFFFFFFKRR & $-^{e}$ & - & $-^{e}$ & - \\
\hline S. mitis-CSP-2-N7II8FF12L & EIRQTHIFFFNLFKRR & 891 & $793-1001$ & $-^{e}$ & - \\
\hline S. mitis-CSP-2-I2MN7IN11F & EMRQTHIIFFFFFKRR & 568 & $429-750$ & $-^{e}$ & - \\
\hline S. mitis-CSP-2-N7FI8FN11F & EIRQTHFFFFFFFKRR & $-^{e}$ & - & $-^{e}$ & - \\
\hline
\end{tabular}

${ }^{a}$ See the Experimental section for full experimental details and the ESI for plots of agonism dose response curves. ${ }^{b} \mathrm{EC}_{50}$ values were determined by testing peptides over a range of concentrations. ${ }^{c} 95 \%$ confidence interval. ${ }^{d} \mathrm{EC}_{50}$ values of $S$. pneumoniae $\mathrm{CSP}$ and $\mathrm{CSP} 2$ from ref. $27 .{ }^{e} \mathrm{EC}_{50}$ not determined due to the analogue's low induction in the primary agonism screening assay.* Key analogues discussed in the text are italicized.

Table $4 \quad I C_{50}$ values of the S. mitis-CSP-2-E1A modification analogues against the S. pneumoniae ComD1 and ComD2 receptors ${ }^{a}$

\begin{tabular}{|c|c|c|c|c|c|}
\hline \multirow[b]{2}{*}{ Peptide name } & \multirow[b]{2}{*}{ Peptide sequence } & \multicolumn{2}{|c|}{ S. pneumoniae ComD1 } & \multicolumn{2}{|c|}{ S. pneumoniae ComD2 } \\
\hline & & $\mathrm{IC}_{50}^{b}(\mathrm{nM})$ & $95 \% \mathrm{CI}^{c}$ & $\mathrm{IC}_{50}^{b}(\mathrm{nM})$ & $95 \% \mathrm{CI}^{c}$ \\
\hline S. pneumoniae CSP1-E1A ${ }^{e}$ & AMRLSKFFRDFILQRKK & 85.7 & $50.8-145$ & $-^{d}$ & $-^{d}$ \\
\hline S. mitis-CSP-2-E1A & AIRQTHNIFFNFFKRR & 497 & $422-585$ & $-^{d}$ & - \\
\hline S. mitis-CSP-2-E1AI2M & AMRQTHNIFFNFFKRR & 85.4 & $57.4-127$ & $-^{d}$ & - \\
\hline S. mitis-CSP-2-E1AN11F & AIRQTHNIFFFFFKRR & $-^{d}$ & - & $-^{d}$ & - \\
\hline S. mitis-CSP-2-E1AI2MQ4L & AMRLTHNIFFNFFKRR & 54.2 & $40.6-72.2$ & $-^{d}$ & - \\
\hline S. mitis-CSP-2-E1AN11FF12L & AIRQTHNIFFFLFKRR & $-^{d}$ & - & $-^{d}$ & - \\
\hline S. mitis-CSP-2-E1AN7II8F & AIRQTHIFFFNFFKRR & 204 & $133-311$ & 135 & $82.5-222$ \\
\hline S. mitis-CSP-2-E1AI2MF12L & AMRQTHNIFFNLFKRR & $-^{d}$ & - & $-^{d}$ & - \\
\hline S. mitis-CSP-2-E1AI2MI8F & AMRQTHNFFFNFFKRR & 143 & $88.9-229$ & $-^{d}$ & - \\
\hline S. mitis-CSP-2-E1AI2MN7FI8F & AMRQTHFFFFNFFKRR & 294 & $263-328$ & 418 & $235-744$ \\
\hline S. mitis-CSP-2-E1AI2MN7II8F & AMRQTHIFFFNFFKRR & 141 & $81.8-243$ & 32.9 & $16.4-66.0$ \\
\hline S. mitis-CSP-2-E1AI2MQ4LI8F & AMRLTHNFFFNFFKRR & 317 & $238-423$ & $-^{d}$ & - \\
\hline S. mitis-CSP-2-E1AI2MI8FN11F & AMRQTHNFFFFFFKRR & ${ }^{d}$ & - & $-^{d}$ & - \\
\hline
\end{tabular}

${ }^{a}$ See the Experimental section for full experimental details and the ESI for plots of antagonism dose response curves. ${ }^{b}$ IC $_{50}$ values were determined by testing peptides over a range of concentrations. ${ }^{c} 95 \%$ confidence interval. ${ }^{d} \mathrm{IC}_{50}$ not determined due to the analogue's low induction in the primary antagonism screening assay. ${ }^{e} \mathrm{IC}_{50}$ value of $S$. pneumoniae CSP1-E1A from ref. 27. 
both pneumococcal ComD receptors (Table 4). Since the N11F modification resulted in highly potent QS activators, the lack of inhibitory activity for all the E1A-based analogues bearing this modification highlights the stringent and different requirements for ComD receptor inhibition compared to receptor activation. Most importantly, from the $S$. mitis-CSP-2-E1Abased library we identified three nanomolar range antagonists of both pneumococcus pherotypes, $S$. mitis-CSP-2-E1AN7II8F, S. mitis-CSP-2-E1AI2MN7II8F and $S$. mitis-CSP-2-E1AI2MN7FI8F (Table 4). These potent $S$. mitis-CSP-2-based pan-group inhibitors of the pneumococcus competence regulon are an important addition to the arsenal of chemical tools available to study pneumococcal behavior and attenuate pneumococcal infections.

\section{Conclusions}

In conclusion, we set out to investigate potential crosstalk between streptococci species and utilize the identified signaling molecules to develop novel pneumococcal QS modulators. First, our analysis revealed that the majority of CSP signals from closely related streptococci of the mitis group, as well as CSPs from other groups of streptococci, were unable to modulate QS in S. pneumoniae, highlighting the relatively high specificity of the pneumococcus ComD receptors to their cognate native CSP signals. Second, our analysis also revealed a potential role of $S$. mitis as a modulator of the competence regulon in $S$. pneumoniae. Our rationally-designed $S$. mitis-CSP2-based analogues, generated using the SAR results of both the CSP1 and CSP2 scaffolds in $S$. pneumoniae, ${ }^{27}$ yielded several potent pan-group agonists of pneumococcal QS. Moreover, our analysis revealed that by using the $S$. mitis-CSP-2 pheromone as a template, it is possible to develop potent antagonists of the pneumococcal competence regulon. Most importantly, our work revealed the first pan-group $S$. mitis-CSP-2-based ComD inhibitors of $S$. pneumoniae with activities in the nanomolar range.

Since $S$. mitis has the potential to influence the regulation of pneumococcus phenotypes associated with virulence and infectivity, developing highly potent QS modulators using the native CSP pheromone of $S$. mitis, $S$. mitis-CSP-2, with enhanced potency could lead to a complementary strategy to attenuate pneumococcal infections. Furthermore, these privileged scaffolds could influence the competence regulon in $S$. mitis, thus providing novel chemical tools capable of modulating QS in multiple species. Indeed, experiments aimed at identifying the effect of the $S$. mitis-CSP-2 analogues on the competence regulon in $S$. mitis are ongoing in our laboratory and will be reported in due course.

\section{Experimental section}

\section{Chemical reagents and instrumentation}

All the chemical reagents and solvents were purchased from Sigma-Aldrich or Chem-Impex and used without further purification. Water (18 $\mathrm{M} \Omega$ ) was purified using a Millipore
Analyzer Feed System. Solid-phase resins were purchased from Advanced Chem Tech.

Reversed-phase high-performance liquid chromatography (RP-HPLC) was performed using a Shimadzu system equipped with a CBM-20A communications bus module, two LC-20AT pumps, an SIL-20A auto sampler, an SPD-20A UV/vis detector, a CTO-20A column oven, and an FRC-10A fraction collector. The matrix-assisted laser desorption ionization time-of-flight mass spectrometry (MALDI-TOF MS) data were obtained using a Bruker Microflex spectrometer equipped with a $60 \mathrm{~Hz}$ nitrogen laser and a reflectron. In positive ion mode, the acceleration voltage on Ion Source 1 was $19.01 \mathrm{kV}$. The exact mass (EM) data were obtained using an Agilent Technologies 6230 TOF LC/MS spectrometer. The samples were sprayed with a capillary voltage of $3500 \mathrm{~V}$ and the electrospray ionization (ESI) source parameters were as follows: gas temperature of $325{ }^{\circ} \mathrm{C}$ at a drying gas flow rate of $8 \mathrm{~L} \mathrm{~min}{ }^{-1}$ at a pressure of $35 \mathrm{psi}$.

\section{Peptide synthesis}

All the CSP analogues were synthesized using the standard Fmoc-based solid-phase peptide synthesis (SPPS) procedures on 4-benzyloxybenzyl alcohol (Wang) resin. Pre-loaded Fmoc-L-Arg (Pbf) Wang resin (0.305 mmol $\left.\mathrm{g}^{-1}\right)$, Fmoc-L-Lys (Boc) Wang resin $\left(0.59 \mathrm{mmol} \mathrm{g}^{-1}\right)$, or Fmoc-Asn (Trt) Wang resin $\left(0.332 \mathrm{mmol} \mathrm{g}^{-1}\right)$ was used for peptides that required an arginine, lysine, or asparagine at the $C$-terminus, respectively. CSP and analogues were synthesized by using the Liberty 1 automated peptide synthesizer (CEM Corporation) (for the full procedures, see the ESI $\dagger$ ).

\section{Peptide purification by HPLC}

Crude peptides were purified with RP-HPLC. The crude peptide was dissolved using $\mathrm{ACN}: \mathrm{H}_{2} \mathrm{O}(1: 3)$ (volume of ACN in water depends on the solubility of the peptide) and purified in $5 \mathrm{~mL}$ portions on a semipreparative Phenomenex Kinetex C18 column $(5 \mu \mathrm{m}, 10 \mathrm{~mm} \times 250 \mathrm{~mm}, 110 \AA)$ with a $5 \mathrm{~mL} \mathrm{~min}{ }^{-1}$ flow rate; mobile phase $\mathrm{A}=18 \mathrm{M} \Omega$ water $+0.1 \%$ TFA and mobile phase $\mathrm{B}=\mathrm{ACN}+0.1 \%$ TFA. The collected fraction was frozen using an acetone/dry ice bath, lyophilized overnight, and dissolved again in $5 \mathrm{~mL}$ ACN : $\mathrm{H}_{2} \mathrm{O}(1: 3)$ for a secondary purification run. Preparative HPLC methods were used to separate the crude peptide mixture to different chemical components using the following linear gradient: (first prep 5\% B $\rightarrow 45 \%$ B over $40 \mathrm{~min}$ and second prep $25 \% \mathrm{~B} \rightarrow 38 \% \mathrm{~B}$ over $45 \mathrm{~min}$ ). Fraction purity was determined through analysis with a Phenomenex Kinetex analytical C18 column $(5 \mu \mathrm{m}, 4.6 \mathrm{~mm} \times 250 \mathrm{~mm}, 110 \AA)$ using a linear gradient ( $5 \%$ B $\rightarrow 95 \%$ B over $27 \mathrm{~min})$. Purities were determined by the integration of peaks with UV detection at $220 \mathrm{~nm}$. Only peptide fractions that were purified to homogeneity ( $>95 \%$ ) were used for the biological assays.

\section{Biological assays}

Biological reagents and strain information. All standard biological reagents were purchased from Sigma-Aldrich and used according to the enclosed instructions. Donor horse serum (defibrinated) was stored at $4{ }^{\circ} \mathrm{C}$ until use in bacterial growth conditions. To examine the ability of the synthesized 
CSP analogues to modulate the ComD receptors, and thus, the QS circuit in S. pneumoniae, beta-galactosidase assays were performed using D39pcomX::lacZ (group I) and TIGR4pcomX::lacZ (group II) reporter strains. ${ }^{26}$

Bacterial growth conditions. Freezer stocks of individual pneumococcal strains, D39pcomX::lacZ and TIGR4pcomX::lacZ, were created from $1.5 \mathrm{~mL}$ aliquots of bacteria $\left(0.2 \mathrm{OD}_{600 \mathrm{~nm}}\right)$ in Todd-Hewitt broth supplemented with $0.5 \%$ yeast extract (THY) and $0.5 \mathrm{~mL}$ glycerol, and stored at $-80{ }^{\circ} \mathrm{C}$. For experiments, bacteria from the freezer stocks were streaked onto a THY agar plate supplemented with $5 \%$ horse serum and chloramphenicol at a final concentration of $4 \mu \mathrm{g} \mathrm{mL} \mathrm{m}^{-1}$. The plate was incubated for $8 \mathrm{~h}$ in a $\mathrm{CO}_{2}$ incubator $\left(37^{\circ} \mathrm{C}\right.$ with $\left.5 \% \mathrm{CO}_{2}\right)$. Isolated fresh colonies were then transferred to $5 \mathrm{~mL}$ of THY broth containing $4 \mu \mathrm{g} \mathrm{mL}{ }^{-1}$ of chloramphenicol and incubated in a $\mathrm{CO}_{2}$ incubator overnight $(15 \mathrm{~h})$. These overnight cultures were then diluted (1:50 for D39pcomX::lacZ; 1:10 for TIGR4pcomX::lacZ) with THY and the resulting solution was incubated in $\mathrm{a} \mathrm{CO}_{2}$ incubator for 3-4 hours, until the bacteria reached the early exponential stage (OD 600 values of $0.30-0.35$ for D39pcomX::lacZ and $0.20-0.25$ for TIGR4pcomX::lacZ) as determined by using a plate reader.

Beta-galactosidase assays. The ability of the synthetic CSP analogues to activate the QS circuit was evaluated as previously described (see the ESI $\dagger$ for full experimental details). ${ }^{27,28}$

$\mathbf{E C}_{50}$ experiments. Analogues that exhibited high activity in the initial screening were further evaluated using a dosedependent assay in which peptide stock solutions were diluted with DMSO in serial dilutions (either 1:2, 1:3 or 1:5) and assayed as described in the ESI. $\dagger$ GraphPad Prism 5 was used to calculate the $\mathrm{EC}_{50}$ values, which are the concentration of drug that gives half-maximal response.

$\mathbf{I C}_{50}$ experiments. Analogues that exhibited low $S$. pneumoniae $\operatorname{com} X$ activation in the initial screening were evaluated for competitive inhibition. The ability of the synthesized CSP analogues to inhibit the expression of $S$. pneumoniae comX by outcompeting $S$. pneumoniae CSP for the receptor binding site was evaluated using the same assay conditions as described in the ESI, $\dagger$ except that in the initial inhibition screening, the native $S$. pneumoniae CSP was added to every well at a set concentration (50 nM $S$. pneumoniae CSP1 for group I; $250 \mathrm{nM}$ $S$. pneumoniae CSP2 for group II) that was chosen to afford full activation of the QS circuit, as determined from the dosedependent curves created for the native S. pneumoniae CSPs. Two $\mu \mathrm{L}$ of native CSP ( $5 \mu \mathrm{M}$ solution of CSP1 for group I; $25 \mu \mathrm{M}$ solution of CSP2 for group II) and $2 \mu \mathrm{L}$ of $1 \mathrm{mM}$ solution of the synthetic CSP analogues were added to the same well in triplicate in a clear 96-well microtiter plate. Two $\mu \mathrm{L}$ of native CSP $(5 \mu \mathrm{M}$ solution of CSP1 for group I; $25 \mu \mathrm{M}$ solution of CSP2 for group II) and $2 \mu \mathrm{L}$ of DMSO were added to the same well in triplicate and served as the positive control. Four $\mu \mathrm{L}$ of DMSO were added in triplicate and served as the negative control. Then, $196 \mu \mathrm{L}$ of the bacterial culture was added to the wells and the plate was incubated at $37{ }^{\circ} \mathrm{C}$ for $30 \mathrm{~min}$. The procedure for lysis, incubation with ONPG and all the measurements was as described in the beta-galactosidase assay section in the ESI. $\uparrow$ Analogues that exhibited significant competitive inhibition in the initial screening were further evaluated using a dose-dependent assay where peptide stock solutions were diluted with DMSO in serial dilutions (either 1:2, 1:3, or 1:5) and assayed as described above. GraphPad Prism 5 was used to calculate the $\mathrm{IC}_{50}$ values, which are the concentration of inhibitor where the response (or binding) is reduced by half.

\section{Conflicts of interest}

The authors declare no competing financial interest.

\section{Acknowledgements}

This work was supported by a grant from the National Institutes of Health (R35GM128651). S. pneumoniae D39pcomX::lacZ and TIGR4pcomX::lacZ reporter strains were generous gifts from G. W. Lau (University of Illinois at Urbana-Champaign).

\section{References}

1 S. Mehr and N. Wood, Paediatr. Respir. Rev., 2012, 13, 258-264. 2 S. S. Huang, K. M. Johnson, G. T. Ray, P. Wroe, T. A. Lieu, M. R. Moore, E. R. Zell, J. A. Linder, C. G. Grijalva and J. P. Metlay, Vaccine, 2011, 29, 3398-3412.

3 J. Cornick and S. Bentley, Microbes Infect., 2012, 14, 573-583. 4 N. J. Croucher, S. R. Harris, C. Fraser, M. A. Quail, J. Burton, M. van der Linden, L. McGee, A. von Gottberg, J. H. Song and K. S. Ko, Science, 2011, 331, 430-434.

5 L. Håvarstein, G. Coomaraswamy and D. A. Morrison, Proc. Natl. Acad. Sci. U. S. A., 1995, 92, 11140-11144.

6 G. W. Lau, S. Haataja, M. Lonetto, S. E. Kensit, A. Marra, A. P. Bryant, D. McDevitt, D. A. Morrison and D. W. Holden, Mol. Microbiol., 2001, 40, 555-571.

7 L. Zhu, J. Lin, Z. Kuang, J. E. Vidal and G. W. Lau, Mol. Microbiol., 2015, 97, 151-165.

8 M. R. Oggioni, C. Trappetti, A. Kadioglu, M. Cassone, F. Iannelli, S. Ricci, P. W. Andrew and G. Pozzi, Mol. Microbiol., 2006, 61, 1196-1210.

9 D. L. Hava and A. Camilli, Mol. Microbiol., 2002, 45, 1389-1406.

10 S. T. Rutherford and B. L. Bassler, Cold Spring Harbor Perspect. Med., 2012, 2, a012427.

11 D. N. McBrayer, B. K. Gantman, C. D. Cameron and Y. Tal-Gan, Org. Lett., 2017, 19, 3295-3298.

12 B. LaSarre and M. J. Federle, Microbiol. Mol. Biol. Rev., 2013, 77, 73-111.

13 Y. Tal-Gan, D. M. Stacy, M. K. Foegen, D. W. Koenig and H. E. Blackwell, J. Am. Chem. Soc., 2013, 135, 7869-7882.

14 Y. Tal-Gan, M. Ivancic, G. Cornilescu, T. Yang and H. E. Blackwell, Angew. Chem., Int. Ed., 2016, 55, 8913-8917.

15 A. Harrington and Y. Tal-Gan, J. Bacteriol., 2018, 200, e00709-e00717.

16 R. W. Mull, A. Harrington, L. A. Sanchez and Y. Tal-Gan, Curr. Top. Med. Chem., 2018, 18, 625-644.

17 O. Johnsborg, P. E. Kristiansen, T. Blomqvist and L. S. Håvarstein, J. Bacteriol., 2006, 188, 1744-1749. 
18 G. Pozzi, L. Masala, F. Iannelli, R. Manganelli, L. Havarstein, L. Piccoli, D. Simon and D. Morrison, J. Bacteriol., 1996, 178, 6087-6090.

19 G. Salvadori, R. Junges, R. Khan, H. A. Åmdal, D. A. Morrison and F. C. Petersen, Methods Mol. Biol., 2017, 1537, 219-232.

20 M. Kilian, K. Poulsen, T. Blomqvist, L. S. Håvarstein, M. Bek-Thomsen, H. Tettelin and U. B. Sørensen, PLoS One, 2008, 3, e2683.

21 L. S. Håvarstein, R. Hakenbeck and P. Gaustad, J. Bacteriol., 1997, 179, 6589-6594.

22 G. Salvadori, R. Junges, D. A. Morrison and F. C. Petersen, Front. Microbiol., 2016, 7, 1009.

23 S. Shekhar, R. Khan, D. M. Ferreira, E. Mitsi, E. German, G. H. Rørvik, D. Berild, K. Schenck, K. Kwon and F. Petersen, Front. Immunol., 2018, 9, 747.

24 C. R. Bikash, S. R. Hamry and Y. Tal-Gan, ACS Infect. Dis., 2018, 4, 1385-1394.

25 W. C. Chan and P. D. White, Fmoc solid phase peptide synthesis: a practical approach, Oxford University Press, Oxford, 2000.
26 L. Zhu and G. W. Lau, PLoS Pathog., 2011, 7, e1002241.

27 Y. Yang, B. Koirala, L. A. Sanchez, N. R. Phillips, S. R. Hamry and Y. Tal-Gan, ACS Chem. Biol., 2017, 12, 1141-1151.

28 B. Koirala, N. R. Phillips and Y. Tal-Gan, ACS Med. Chem. Lett., 2019, 10, 880-886.

29 Y. Yang and Y. Tal-Gan, Bioorg. Chem., 2019, 89, 102987.

30 Y. Yang, G. Cornilescu and Y. Tal-Gan, Biochemistry, 2018, 57, 5359-5369.

31 A. Domenech, A. R. Brochado, V. Sender, K. Hentrich, B. Henriques-Normark, A. Typas and J. W. Veening, Cell Host Microbe, 2020, 27, 544-555 e543.

32 B. Koirala, J. Lin, G. W. Lau and Y. Tal-Gan, ChemBioChem, 2018, 19, 2380-2386.

33 Y. Yang, J. Lin, A. Harrington, G. Cornilescu, G. W. Lau and Y. Tal-Gan, Proc. Natl. Acad. Sci. U. S. A., 2020, 117, 1689-1699.

34 B. Koirala, R. A. Hillman, E. K. Tiwold, M. A. Bertucci and Y. Tal-Gan, Beilstein J. Org. Chem., 2018, 14, 1769-1777.

35 B. Koirala and Y. Tal-Gan, ChemBioChem, 2020, 21, 340-345. 